\title{
Unilateral Neglect: Assessment and Rehabilitation
}

\author{
Richard P. Conti ${ }^{1, *}$, Jacqueline M. Arnone ${ }^{2}$ \\ ${ }^{1}$ School of Psychology, Kean University, United States \\ ${ }^{2}$ School of Nursing, Kean University, United States
}

Copyright $\bigcirc 2016$ by authors, all rights reserved. Authors agree that this article remains permanently open access under the terms of the Creative Commons Attribution License 4.0 International License

\begin{abstract}
Over 795,000 individuals in the U.S. suffer from strokes annually, with 610,000 accounting for first attacks. Stroke represents the fifth leading cause of death and resulting long-term disability in the United States. Unilateral neglect (UN) is a disabling characteristic of stroke, occurring from lesion damage to one hemisphere of the brain. Individuals with UN do not respond normally to objects, stimuli, and people located to their contralesional side. Right hemispheric lesions exhibit the greatest impairment. Incidence rates of UN vary from 13\%-81\%. Impairment of this condition impedes an individual's capacity to execute activities of daily living. Diagnosis can be challenging and if left untreated, results in poorer patient outcomes. While standardized test battery covering multiple domains have demonstrated enhanced detection for diagnosis over single test methods, and combination treatment modalities have shown promise in procuring symptom abatement, there is a paucity of evidence on the long-term effects of neglect therapy and its transferability into daily life. Stroke expenditures impose a significant economic encumbrance on healthcare systems with costs estimated at $\$ 34$ billion per annum in the U.S. alone. Effectively delineating and treating UN would enhance integrated stroke services, improve patient outcomes and reduce the lifetime cost of stroke.
\end{abstract}

Keywords Unilateral Neglect, Stroke Treatment, Stroke Rehabilitation, Activities of Daily Living, Economic Burden

\section{Introduction}

In many countries stroke affects up to $0.3 \%$ of the population annually [1] and is the second most common cause of death [2]. In the United States, stroke represents the fifth leading cause of death and accounts for a significant source of prevailing disability among affected individuals [3]. Each year, more than 795,000 individuals in the United States suffer from stroke, with 610,000 accounting for first or new strokes [3]. It is estimated that by 2030 , an additional 3.4 million people will suffer a stroke, a $20.5 \%$ increase in prevalence from 2012 data [4].
Moreover, stroke expenditures in the United States are an estimated \$34 billion annually [4]. This sum includes the charges for health care services, prescriptions to treat stroke, and lost productivity due to morbidity and mortality $[3,5]$.

Unilateral Neglect (UN) is a disabling characteristic of stroke, as a result of lesion damage to one hemisphere of the brain [6,7]. This disorder is a multifaceted and complex neurological condition in which an individual fails to respond to stimuli in the contralesional hemispace, which cannot be explained by primary sensory or motor defects $[8,9]$. Right hemispheric lesions exhibit the greatest impairment $[10,11]$ and incidence rates vary from $13 \%-81 \%$ [12-14]. UN is also more severe in older patients [10]. Research on gender differences in UN have produced mixed results. A Nigerian study of 40 post-stroke patients found that neglect was present in $66.7 \%$ of females compared to $13.6 \%$ of males [15]. Conversely, Kleinman et al.[16] found no gender differences in the prevalence or severity of neglect within 24 hours of ischemic stroke in 312 right-handed patients, which supports earlier findings [17-18]. McGlone et al. [17] reported no significant differences between genders in predilection and severity of symptoms of neglect after unilateral stroke from either right or left hemisphere lesions. Results were based on copy/drawing, line bisection, and figure and line cancellation tasks. Interestingly, the incidence was found to be greater in women with right-sided lesions when the neglect was centered on scores amid contralesional versus ipsilateral response times on a Visual Search Task. Kleinman et al. [16] suggest there may not be a difference between genders in either frequency or severity of neglect noting that previous studies may be inconclusive due to the, "limited task number, time frame (post stroke) of testing, small sample size, mean age within each gender group, variability of lesion volume, or any combination of these factors" (p. 2).

Neglect is seen most frequently in patients who have experienced right hemisphere damage (usually due to stroke, but sometimes neglect may coincide with a traumatic brain injury) $[14,19,20]$. The presentation of neglect is not limited to the right hemisphere. It has also been documented to occur with left hemispheric lesions in which individuals yield to neglect rightward. Left hemispheric lesions are typically less 
severe and shorter in duration than right hemispheric damage and neglect [11]. However, UN may be temporary and reversible when it occurs in conjunction with seizures, electroconvulsive therapy, and intracarotid sodium amytal testing (Wada testing) $[9,21,22]$.

Individuals who experience neglect typically do not respond to stimuli located in the contralesional space. For example, patients with right hemispheric neglect may not eat the food on the left side of their plate, shave only the right side of their face, fail to read words on the left-hand side of a page, or when asked to draw an object, are only able to reproduce the right side [21-23]. While activities of daily living (ADL) can become hindered [24], there are more serious consequences associated with UN. Safety issues can arise and become problematic in this population due to potential falls, crossing the street and driving a vehicle [25]. Social behavior may also be impacted as a result of social-emotional deviations including changes in personality and affect [26]. Furthermore, research has indicated that the presence of neglect in stroke patients is considered an adverse indicator of their prognosis for recovery [22,24,27].

\section{Unilateral Neglect}

$\mathrm{UN}$, also referred to in the literature as hemineglect [28], hemispatial neglect [29], spatial neglect [30], visual neglect [31], visual spatial neglect [32], inattention [21] and unilateral spatial neglect [33], is among one of the most disabling, prominent and long lasting impairments of the right hemisphere following stroke, whereby affected individuals demonstrate failure in spatial awareness of objects to one side [34,35]. Jepson et al. [36] report that UN occurs, as a result of stroke, in as many as 200,000 individuals in the United States, and largely hinders the skills required to function in everyday life [37]. Additionally, Edwards et al. [38] report that of approximately 3 million stroke survivors in the US, $70 \%$ encounter salient functional disability in mobility, ADL, social integration, and gainful employment.

UN can occur in both the acute and chronic phase after a stroke, and presentation of symptoms can attenuate over time with regards to the level of impairment and disability [39]. Characteristically, researchers have associated this syndrome with injury to the right posterior parietal cortex of the brain, but contemporary studies have contested this interpretation, citing additional neural loci may be implicated [40,41]. Additionally, these disparities of both location and lesion size might influence the observed heterogeneity of the disorder [41].

According to Zillmer and Spiers [42] in UN there is conscious inattention to the left side with a shift in awareness to the right. Although UN can occur after damage to either hemisphere, left-side neglect due to right-hemisphere damage is far more common, longer lasting, and more severe than right-side neglect due to left-hemisphere damage $[16,21,40,44]$.
Spontaneous recuperation has been observed to occur partially or in totality within the first month after stroke [44]. Though Farné et al. [39] report left-visual spatial neglect can remain acute in numerous individuals and unceasingly persevere. The existence of the condition appreciably hinders hemiparesis recovery and activities of daily living can become problematic for the individual affected [45]. In some cases, individuals with severe UN fail to make eye movements towards stimuli on the contralesional side. Instead, their eyes deviate towards the ipsilesional side, (i.e., the side with the lesion) [46-48]. However, the UN response is not due to any loss of vision; instead it is a cognitive inability to perceive what is in the visual field on the contralesional side.

According to Mesulam [49], "neglect is not a defect of seeing, hearing, feeling, or moving but one of looking, listening, touching, and searching" (p. 142). Although neglect may be demonstrated in more than one modality, it is usually confined to the visual performance, indicating a significant relationship between spatial attention and movement [24,45,50]. Kerkhoff and Schenk [24] state early efforts to manage patients were directed towards visual impairment, which is the most evident clinical presentation, yet research over the past 20 years has provided greater clarity into both the spatial and non-spatial processes that result in neglect manifestation [23]. Given the multiple intervention methods used over the past 60 years directed at decreasing the consequences of left visual-spatial neglect, the short-term effects noted have yet to be proven conclusive with regard to long-term functional outcomes for this population [34].

\section{Attentional Deficits}

Unilateral neglect is subdivided into three key classifications of attentional deficits [44]. These categories include: (a) memory and representational deficits; (b) action-intentional conditions (motor neglect); and (c) inattention (sensory neglect). A brief overview of each is subtype is detailed below.

\subsection{Motor Neglect}

Individuals who are suffering from motor neglect typically experience an absence of spontaneous movement in the contralesional limb, which is not explained by damage to the motor cortex [46]. For instance, when individuals are asked to raise both hands, they frequently only elevate the hand ipsilateral to the lesion site [50]. It is important to note that motor neglect involves the contralateral side of the body and not the contralateral environment, as is represented in the typical clinical presentation of unilateral spatial neglect. The occurrence of motor intentional neglect can range from $6 \%$ to over $40 \%$ of patients with $\mathrm{UN}$, and rates are contingent upon what way poor motor intention is operationally defined [29]. 


\subsection{Neglect Dyslexia}

Patients with neglect dyslexia will characteristically fail to read the left part of sentences or single words [11,51]. For example, "smile" is read as "mile" or "belief" as "grief." Omissions usually consist of the initial letter or substitutions of the original letter to form a grammatically correct word. The opposite effect has also been reported with left hemisphere lesions [11]. For example, "south" is read as "soup" or "modern" as "modest." When asked to read a complete sentence or a passage, patients often begin reading at the middle of the sentence and read the entire passage in this manner despite the meaningless of the text $[11,51]$.

\subsection{Neglect Dysgraphia}

The symptoms of neglect dysgraphia usually involve a preference for the right page of a document, which causes the individual to produce crowded handwriting with an inflated left margin [21]. It is certainly not out of the ordinary for people with neglect dysgraphia to have difficulty writing horizontally, as well as leaving large spaces between words and committing graphic errors [52]. In both neglect dysgraphia and neglect dyslexia the pattern of errors may vary widely between patients [21]. It is important to note that both conditions do not always co-occur [53,54]. In a study of neglect dyslexia, Primativo et al. [55] found that UN patients rendered left-lateralized exclusion errors as a result of the simultaneous presence of both neglect and distorted oculomotor patterning. According to the researchers, "preventing the automatic execution of the fine saccadic eye movements involved in reading, uncovers the attentional bias also in reading single centrally presented words" (p. 2109).

\subsection{Facial Neglect}

Patients with facial neglect show a defect in the recognition of normal faces and may only recognize half faces [56]. Young et al. [57] describe this as a domain-specific form of $\mathrm{UN}$ and found the deficit was present with the representation of both internal and external facial features. Research has also indicated that in facial neglect deficits are also found in the judgment of facial expressions and the resemblance between faces [56,57]. It has been suggested that facial neglect occurs from a failure to attend to the left side of faces, due to the injury of the right hemisphere that produces a right-sided cognitive preference [57].

\subsection{Auditory Neglect}

Although a few studies have reported the presence of auditory neglect, it remains complicated to validate $[58,59]$. This difficulty arises from the fact that the auditory system is not nearly as lateralized as the visual system. Therefore, testing with unilateral stimuli cannot be accurately controlled.
For instance, past studies have utilized the presentation of bilateral auditory stimulus and defined the failure to respond to the stimulus in the left hemispace as auditory neglect $[21,60]$. Due to the bilateral nature of the auditory system, this form of neglect is often referred to as inattention. This type of deficiency in aural functioning is also known as extinction; however, it has been argued that extinction is nothing more than an attenuated form of neglect [50].

\section{Related Disorders and Causation}

UN has also been associated with a number of other disorders. These include: (a) anosognosia--the loss of ability to recognize or to acknowledge an illness or body defect [61]; (b) anosodiaphoria--an awareness of the contralateral defect despite an apparent lack of concern about the impairment [62]; (c) allesthesia or allochiria--a failure to detect unilateral stimuli [54]; (d) extinction--a failure to identify both stimuli in the presentation of bilateral simultaneous presentation [50,54]; and (e) emotional dysregulation-disorders of emotional processing [63].

\subsection{Anosognosia}

Anosognosia refers to the loss of ability to recognize or to acknowledge an illness or body defect [60], and is a critical prognosticator of poor rehabilitative outcome [61,64]. Neglect and anosognosia occur in mostly right frontal hemispheric stroke with lesions found in the parietal-temporal-occipital area [64]. Patients are typically older than traditional UN patients, exhibit pre-stroke dementia, and have larger lesion sites [61]. The existence of anosognosia may associate with treatment failure, especially if the selected therapeutic modality utilizes strategic or top-down measures [63]. Anosognosia may influence the patient's engagement in learning [65] and it has been associated with impairments of cognition and subcortical brain atrophy [26].

\subsection{Extinction}

Extinction is defined as a failure to identify both stimuli in the presentation of bilateral simultaneous presentation $[50,54]$. Not all patients who present with extinction have neglect, and it has been suggested that extinction is a less severe form of neglect, or an indication of inattention [66]. Parton et al. [66] point out that extinction is not only localized to one mode; it may also be observed in tactile, auditory, and cross-modal form.

\section{Neural Structures Affected}

The posterior region of the inferior parietal lobe in the right hemisphere is most often implicated in UN [21,54]. However, UN has also been associated with damage to 
subcortical structures including the thalamus. These areas are interrelated and believed to comprise an important "neural circuit." Therefore, damage to any one of them may affect the functioning of the others $[9,52]$.

\section{Theories of UN Based on the Cause of Symptoms}

According to Smith et al. [37], "although unilateral neglect has received a great deal of attention in the literature in recent decades; no single theory has been able to account for all aspects of this deficit" (p. 29). Presently, there are two general types of theories regarding the cause of UN symptoms: Attention theories and representational theories [54]. In viewing $\mathrm{UN}$ as a disorder of attention, patients are unable to disengage their attention from the side that is ipsilateral to the lesion. Representational theories focus on the disruption of the internal representation of space, after the stimuli have already been perceived by the individual.

\subsection{Awareness Theories and Attentional Orienting}

Advocates of awareness theories hold that individuals with UN have damage to areas of the brain that control attention orienting [54]. For example, individuals with UN focus their attention on the ipsilesional space and fail to shift their attention to the contralesional space, without notice to stimuli on that side. Some proponents of attention theories believe that damage occurs to a neural circuit that generates saccadic (fast eye) movements, which are an important component of visual attention.

\subsection{Representational Theories and Spatial Representation}

Representational theories of UN suggest that patients are unable to construct a complete mental representation of space in the contralesional space. In this case the damage is believed to be to a neural circuit that controls spatial representation and conscious awareness in the brain. Within these two general theories, there are a variety of opinions regarding the specific mechanisms and brain functions that account for the symptoms seen in individuals with $\mathrm{UN}$, which can vary in severity and scope $[50,67,68]$. For example, different sensory modes (visual, auditory, and tactile) and spatial dimensions (horizontal, radial, and vertical) can be affected to different degrees. In fact, neglect symptoms may be task specific, which may create difficulty in individual awareness and therapy $[21,69]$.

\section{Assessment/Testing}

The variability of symptoms in UN make initial the diagnosis problematic [70] and may contribute to UN being overlooked [36]. UN leads to lengthier hospital stays, increases the risk of falls and harm, and ultimately the patient's return to independence $[26,70]$. Menon-Nair et al. [71] conducted a retrospective study of UN among Canadian hospitals and the issues rehabilitative clinicians face in the assessment, identification, and treatment of the disorder. It was suggested that in order to neutralize the results of neglect, it is imperative that, "rehabilitative professionals be astute at (1) identifying the impairment, (2), assessing patients by using tools with strong psychometric properties, and (3) providing interventions aimed at reducing the impairment and functional sequelae" (p. 2556). The results of the study elucidated elevated problem identification of $\mathrm{UN}$, as well as clinician incongruity in utilizing standardized assessment tools and evidenced-based methods for treatment. The consensus among clinicians is that a standardized assessment is critical in the diagnosis of UN [38]. However, Edwards et al. [38] report that there is still resistance by some clinicians to screen for deficits not immediately visible on cursory examination [38].

Clinical testing in the assessment of this disorder has concentrated on evaluating spatial partiality in visuomotor tasks [63]. The presence of visuospatial UN can be detected using some simple screening tests that are quickly and easily administered at the patient's bedside. The most common are line bisection, figure cancellation, figure copying, and representational drawing tasks [21,54]. Although these tests have demonstrated acceptable reliability and validity for diagnosing UN, they are highly variable in exhibiting sensitivity in detecting varying degrees of UN [63]. Moreover, the time between neurological affront and testing has the potential to effect the sensitivity of tests [63].

\subsection{Line Bisection Test}

The line bisection test is the most widely used diagnostic test in the assessment of UN $[21,68]$. In its most common version, the individual is presented with a thin long horizontal line drawn in the center of a piece of paper and asked to bisect the line, either by pointing to the line's midpoint or by quickly making a mark across the line at midpoint. Typically, patients with left-sided UN bisect the line to the right of the actual midpoint of the line. Likewise, right-sided neglect is associated with a leftward shift in people's mark from the midpoint. In either case, the distance from the actual midpoint (called the displacement) is an indicator of the severity of the patient's neglect $[21,72]$.

Although there is a consensus among clinicians that poor performance on the test is a reliable indicator of the presence of UN, the specific mechanisms for showing the severity or particular manifestations of neglect are less understood [21,54]. Various line lengths, orientations, backgrounds, and positions on the paper have been studies with the line bisection test. Previous research has suggested that all of these variables have "reliable effects on the measured severity of neglect" [73] (p. 187). Other factors such as age, reading habits (left-to-right or right-to-left), viewing distance, gender, and handedness have also been examined on line 
bisection performance.

\subsection{Figure Cancellation Tests}

In the cancellation tests, a person is presented with a paper containing various drawn figures, either short lines, letters of the alphabet, or stars scattered around the page [21]. The patient is asked to draw a line through each figure on the page or, for the letter test, through a particular letter. Typically, patients with left-sided neglect cross out figures on the right side of the page and fail to notice the ones on the left side of the page [27]. Omission of more than $5 \%$ of the figures is considered the usual criterion indicating USN [74].

Studies have also indicated that there is a diagonal bias among UN patients during the performance of cancellation tasks. Stroke patients commonly demonstrate diagonal neglect (a corner-based spatial bias) on cancellation tests by omit targets toward one corner of the page, usually the bottom left quadrant [74]. Cancellation tests assess a patient's ability to ignore distracting stimuli and use visual discrimination. Since letters are more complex and require greater control over information processing, the letter cancellation test is considered the most demanding of the three tests and has the greatest distraction factor [27].

Another visual inattention test with a high distraction factor is the balloons test [21]. In this cancellation test, the patient must search and cross out particular shapes scattered amongst a background of very similar and distracting shapes such as circles and circles with a line adjoining (balloons). This test battery can distinguish between attention and visual field deficits.

Omission and repetitive behavior is commonly observed in patients with UN. A study by Keller et al. [75] assessed stimulus-dependent modulation of visual neglect in a touch screen cancellation task. The study examined how distracters and visual feedback impacted the amount of exclusions and perseverations patients affected with $\mathrm{UN}$ made during testing. Using 18 patients with UN matched against 18 healthy controls, participants executed four disparate cancellation tasks on an iPad computer using the following criteria: no feedback (unchanging screen post touching screen), visual feedback (touched screen resulted in color change), visual feedback with distracters $(20$ distracters embedded uniformly in the screen and identified objects changed colors), and vanishing targets (touched objects vanished from the screen). Results indicated that visual feedback and the existence of distracters exacerbated neglect, which is consistent with damaged disconnection from the ipsilateral margin. Incorporation of both spatial attention orienting and brief actions (that rouse each hemibody in distinct sittings) to normal neurological examinations, could not only elevate diagnostic accuracy of somatosensory deficits, but could also be beneficial in forecasting rehabilitation measures for patients with UN [75]. Keller et al. contend that these additional examination measures are not routinely utilized in practice nor mentioned in any of the current clinical textbooks.

\subsection{Copying and Drawing Tests}

In copying tests the patient the patient is asked to copy a simply drawn figure such as a daisy or geometric shape [21]. Patients with left-sided UN typically copy only the right side of the figure, leaving the left side of their drawing blank, unfinished, or distorted in some way. Ishiai et al. [76] found that UN patients shown a complete drawing of a daisy (a large central circle surrounded by many side-by-side smaller circular petals) correctly identified the figure as a daisy and did not mention any missing petals. However, when asked to copy the daisy, the patients consistently drew a daisy that was correct on the right side only, with large gaps between petals on the left side. Interestingly, the same patients were able to arrange a large circle and many smaller circles into the shape of a daisy.

Drawing tests have the patient draw a simple diagram of a common object such as a clock or daisy from memory. In the clock drawing test, the patient is asked to draw the face of a clock putting in all 12 numbers [21,77]. Patients with left-sided UN may confine their drawings to the right side of the paper and distort or even omit the left side of the clock in their drawings placing all 12 numbers on the right side of the clockface [21]. The daisy drawing test requires the patient to draw a daisy and the examiner notes the position of the petals. As in the clock drawing test, patients with $\mathrm{UN}$ may place all of the petals on the right side of the drawing [21].

\subsection{Combination Tests}

Multiple testing has shown to exhibit greater sensitivity than single testing, which most researchers now favor in assessing patients for UN [63]. A review of the literature by Jepson et al. [36] found the current emergency stroke scales commonly used are devoid of indices in measuring UN. It was also reported that the National Institute of Health Stroke Scale does encompass measures to elicit UN; however, the language-dependent part of the scale overshadows other measures. Subtests of a standard neuropsychological test battery, the Behavioral Inattention Test, incorporates 6 tests (line crossing, letter cancellation, star cancellation, figure copying, line bisection and representational drawing) is used to test for UN in patients who have experienced brain injury $[78,79]$. Advantages of this test include adequate validity and reliability with a cutoff total signifying the juncture to cease further testing [67].

Other combination tests include the Naturalistic Action Test that varies in degree difficulty and includes a lateralized attention scale (to detect impairment in that area) and also includes tasks of ADL $[63,80]$. Lastly, the Catherine Bergego Scale (CBS), originally designed for occupational therapists, has shown exceptional reliability and validity in assessing UN [36]. This 4 point Likert-type scale rates neglect on 10 behavioral tasks (i.e., circumnavigating furniture, grooming and eating off a dish) and may show greater sensitivity to pathological spatial deficits than conventional paper and pencil tests [81]. One limitation of 
the CBS is that occupational therapists only utilize the test for brief periods, versus over the span of an entire day [36]. Since UN has shown to be extremely variable in presentation at any given time, subtle changes in the patient's status may not be observed by the rehabilitation clinician at a single point in time [66].

\section{Rehabilitation}

Research indicates that the symptoms of UN will typically stabilize two months after a stroke event [21,54]. In some cases the condition may resolve itself, however in most instances the effects are enduring. UN has been associated with poor performance of daily motor activities, such as writing, poor sitting balance, crossing the street, or recognizing an object that exits on the contralesional side of the visional field. Studies have shown that as UN improves so will functional motor skills. Unfortunately, there has been less than an optimal response to the various therapies, due to the inability to maintain the positive effects of the various treatments that are currently available $[22,28,82]$.

The symptoms of UN are heterogeneous, which may affect an individual's limb movement, vision, hearing, or balance. In some instances, the neglect may be task-specific, such as the involvement of inattention to far away space when attempting to cross the street [21]. Due to this neurological phenomenon, the rehabilitation programs are various and typically involve a top-down or bottom-up approach to therapy. The top-down technique focuses on improving both the perceptional and behavioral biases by stimulating the individual's higher cognitive levels, through such methods as visual scanning. The bottom-up practice is an attempt at modifying the sensorimotor level by initiating passive sensory stimulation, as is used in Transcutaneous Electrical Nerve Stimulation [50,52,69].

\subsection{Constraint and Bilateral Therapies}

Constraint - Induced Movement Therapy (CIMT) is a cognitive method of therapy that involves the conscious protecting of the unaffected side as a means of training the contralesional side to respond with increased normality [50]. In contrast, Bilateral Movement Training (BMT) encourages the individual to utilize the unaffected limb or body side as a technique to effectively guide the affected bodily area [83].

According to the guidelines, these lower level cognitive exercises aid in the development of bilateral activities that include coordination, postural control, and gait. However, studies have indicated that this particular therapy is difficult when the individual is involved in bilateral environmental conditions, such as crossing the street when road traffic is present. It is hypothesized by many researchers that compensation by the patient's ipsilateral side is compromised due to the increased stimuli to which the person must respond in a more complex environment [50].

\subsection{Transcutaneous Electrical Nerve Stimulation (TENS)}

TENS employs electroanalgesia (low-voltage electrical current for treatment of pain) and has been used as a coadjutant therapy in neurorehabilitation for a variety of disorders such as neuropathic pain in spinal cord injury [84], urinary symptoms caused by neurological disease [85], trigeminal and postherpetic neuralgia, and low back pain [86]. In cases of UN, TENS involves the application of nerve stimulation to the contralesional side of the neck, in order to improve balance and restore a symmetric gait in patients. The TENS bottom-up therapy method has received contradictory scholarly reviews, with regard to the technique's effectiveness and patient comfort [86].

According to Punt and Riddoch [50], TENS has the ability to dramatically improve the balance in people suffering from neglect. In addition, these researchers found that if patients directed their vision towards the contralesional side during the treatment their gait showed a significant improvement in symmetry. Those researchers who favor TENS assert that balance is enhanced by improving the activation of the neurological processor that is injured; thereby allowing the undamaged side to function more efficiently. On the other hand, Wenman et al. [22] found patients who received electrical stimulation in combination with self-instructional training showed no benefit from the treatment. In addition, the therapy was not well tolerated by a least two of the participants. Further research [82] has suggested that TENS has the ability to temporarily improve or worsen the symptoms in UN. According to Pizzamiglio et al. [82], eight weeks of TENS treatment did not result in positive changes for all participants.

\subsection{Visual and Spatial Scanning}

The procedures used for visual and spatial scanning therapy are considered to be higher cognitive training that utilizes a top-down approach to treatment [28]. The technique that is utilized encourages the patient to actively and sequentially scan various parts of a simulated visual field, in order to produce the answers to various questions. These inquiries may include information pertaining to: Reading, copying, copying of line drawings on a dot matrix, and figure description.

Pizzamiglio et al. [28] found that the group benefited from the therapy overall; however, individual differences were reported. It was hypothesized that treatment duration or lesion size and dimension may have been factors in these recorded differences. Follow-up interviews with the patients indicated improved scanning ability many months after the therapy was discontinued. Throughout the treatment process participants exhibited significant attitude changes relating to their disability. For instance, when patients noticed improvement in their respective skills they became more aware of the deficit, which aided them in their ability to create compensatory tactics. In addition, the therapy allowed 
patients to actively participate in the rehabilitation process, which appeared to contribute to expressed feelings of positive self-worth and efficacy. It is important to note that some of the patients who were initially indifferent to the disability experienced some depression when they began to understand the severity of impairment.

\subsection{Virtual Environment Training System}

This newly developed bottom-up approach to therapy was created to assist individuals who suffer from UN [8]. This technology aids the patient in everyday tasks, such as crossing the street. The virtual reality equipment encourages attention to the contralesional visual area by creating visual cueing. The theory behind this system is to enable patients with spatial neglect to use their ipsilateral sight as compensation for the injury in the contralesional area. Through training this technology has the ability to reduce asymmetry between the left and right side in cases of UN. In a three-month follow-up study, the positive effects of the training remained, although there was a small degree of neglect symptom increase in some patients. It has been suggested that this method of training is more beneficial than traditional therapies [8]. For instance, scanning therapy must be provided in a variety of living situations for the method not to become task-oriented, which would inhibit the actual improvement of neglect. Conversely, the virtual reality therapy permits flexible usage in the least restrictive environment, which aids the patient in using this method as a way of creating independence when performing the functions of everyday life.

\subsection{Prism Therapy}

Prismatic Adaptation (PA) may significantly reduce the behavioral deficits of neglect, using a bottom-up process. Rode et al. [52] used prisms that displaced the visual field by $10^{\circ}$ in a rightward direction. After a two to five minute exposure to the prism, manual straight ahead pointing shifted significantly to the left. Prior to the prism therapy patients were identified as manually pointing $9^{\circ}$ to the right, resulting in a $70 \%$ compensation for the $10^{\circ}$ optical displacement. Six of the patients who were exposed to PA showed significant improvement in symptoms for both spatial and object neglect. In addition, PA has been found to improve visual-spatial deficits, such as spatial dysgraphia (right page preference, graphic errors, and failure to write horizontally), as well as audio insufficiency. In fact, PA appears to aid in the ability to create and investigate symmetrical inner representations.

Equally impressive is the length of improvement time that is offered by this therapy. Research has indicated that a five-minute session lasts much longer than any other form of therapy. In fact, there have been case studies showing a marked improvement for as long as four days. Further research has demonstrated that repeated sessions of PA exposure, such as two times daily for a period of two weeks, will bring about noteworthy improvement that lasts for a five week period [52].

Researchers are unsure whether PA is a recovery therapy or a form of selective compensation. However, it has been hypothesized that PA may activate the network between the cerebellum, thalamus, and cortical areas of the brain, causing the positive effects of PA therapy. More importantly, PA appears to be the only treatment that focuses on the right cortical hemisphere, as opposed to the traditional training that stimulates the ipsilesional side of the brain [52].

\section{Conclusions}

The mysteries surrounding UN are numerous due to the heterogeneity of the disorder, with various combinations of deficit occurring in different patients [66]. It is not uncommon to have difficulty in diagnosing UN because it is often compensated for by the patient, it may be modality specific, or its existence denied. In many cases, individuals who suffer from neglect will respond accurately to unilateral stimuli while missing stimuli that is contralesional during bilateral representations. Due to these complications, a full battery of testing should be implemented [63] to recognize the severity of the disorder, and to consider treatment options.

\section{REFERENCES}

[1] A. G. Thrift, D.A. Cadilhac, T. Thayabaranathan, G. Howard, V. J. Howard, P. M. Rothwell, G. A. Donnan. Global stroke statistics, International Journal of Stroke, Vol. 9, 6-18, 2014.

[2] R. Lozano, M. Naghavi, K. Foreman, S. Lim, K. Shibuya, V. Aboyans. Global and regional mortality from 235 causes of death for 20 age groups in 1990 and 2010: a systematic analysis for the global burden of disease study 2010. Lancet, Vol. 380, 2095-2128, 2012.

[3] CDC, NCHS. Underlying Cause of Death 1999-2013 on CDC WONDER Online Database, released 2015. Data are from the Multiple Cause of Death Files, 1999-2013, as compiled from data provided by the 57 vital statistics jurisdictions through the Vital Statistics Cooperative Program. Online available from http://www.cdc.gov/stroke/statistics_maps.html

[4] D. Mozaffarian, E. J. Benjamin, A. S. Go, D. K.Arnett, M. J. Blaha, M. Cushman, S. de Ferranti, J. P. Després, H. J. Fullerton, V. J. Howard, M. D. Huffman, S. E. Judd, B. M. Kissela, D. T. Lackland, J. H. Lichtman, L. D. Lisabeth, S. Liu, R. H. Mackey, D. B. Matchar, D. K. McGuire, E. R. Mohler, C. S. Moy, P. Muntner, M. E. Mussolino, K. Nasir, R. W. Neumar, G. Nichol, L. Palaniappan, D. K. Pandey, M. J. Reeves, C. J. Rodriguez, P. D. Sorlie, J. Stein, A. Towfighi, T. N. Turan, S. S. Virani, J. Z. Willey, D. Woo, R. W. Yeh, M. B. Turner. Heart disease and stroke statistics-2015 update: a report from the American Heart Association, Circulation, Vol. 131, e29-e322, 2015.

[5] B. M. Demaerschalk, H. M. Hwang, G. Leung. US cost burden of ischemic stroke: A systematic literature review, The American Journal of Managed Care, Vol. 16, No. 7, 525-533, 2010. 
[6] L. J. Buxbaum. On the right (and left) track: twenty years of progress in studying hemispatial neglect, Cognitive Neuropsychology, Vol. 23, No. 1, 184-201, 2006.

[7] M. Corbetta, M. J. Kincade, C. Lewis, A. Z. Snyder, A. Sapir A. Neural basis and recovery of spatial attention deficits in spatial neglect, Nature Neuroscience, Vol. 8, No. 11, 1603-1610, 2005.

[8] J. Kim, K. Kim, D. Y. Kim, W. H. Chang, C. Park, S. H. Ohn, K. Han, J. Ku, S. W. Nam, I. Y Kim, S. I. Kim. Virtual environment training system for rehabilitation of stroke patients with unilateral neglect: crossing the virtual street, Cyber Psychology and Behavior, Vol.10, 7-15, 2007.

[9] B. Kolb, I. Whishaw I. Fundamentals of human neuropsychology (7th ed.). Worth, New York, 2015.

[10] R. F. Gottesman, J. T. Kleinman, C. Davis, J. Heidler-Gary, M. Newhart, V. Kannan, A. E. Hillis. Unilateral neglect is more severe and common in older patients with right hemispheric stroke, Neurology, Vol.71, No.18, 1439-1444, 2008.

[11] G. Vallar . Spatial neglect, Balint-Homes' and Gerstmann's syndromes, and other spatial disorders, CNS Spectrums, Vol. 12, No.7, 527-536, 2007.

[12] A. Bowen, K. McKenna, R. C. Tallis. Reasons for the variability in the reported rate of occurrence of unilateral neglect after stroke, Stroke, Vol. 30, 1196-1202, 1999.

[13] K. N. K. Fong, N. Y. H. Yang, M. K. L. Chan, D. Y. L. Chan, A. F. C. Lau, D. Y. W. Chan, J. T. Y. Cheung, H. K. Y. Cheung, R. C. K. Chung, C. C. H Chan. Combined effects of sensory cueing and limb activation on unilateral neglect in subacute left hemiplegic stroke patients: A randomized controlled pilot study, Clinical Rehabilitation, Vol. 27, No.7, 628-637, 2013

[14] S. P. Stone, P. Patel, R. J. Greenwood, P. W. Halligan. Measuring visual neglect in acute stroke and predicting its recovery: the visual neglect recovery index. Journal of Neurology, Neurosurgery, and Psychiatry, Vol. 55, 431-436, 1992.

[15] T. K. Hamzat, S. Y. Oyedele, G. O. Peters. Clinical and demographic correlates of unilateral spatial neglect among Community-dwelling Nigerian stroke survivors, African Journal of Neurological Sciences, Vol. 31, No. 1, 2012.

[16] J. T. Kleinman, M. Newhart, C. Davis, J. Heidler-Gary, R. F. Gottesman, A. E. Hillis. Right hemispatial neglect: frequency and characterization following acute left hemisphere stroke, Brain and Cognition, Vol. 64, 50-59, 2007.

[17] J. McGlone, B. J. Losier, S. E. Black. Are there sex differences in hemispatial visual neglect after unilateral stroke? Neuropsychiatry, Neuropsychology, and Behavioral Neurology, Vol.10, No. 2, 125-134, 1997.

[18] J. M. Ringman, J. L. Saver, R. F. Woolson, W. R. Clarke, H. P. Adams. Frequency, risk factors, anatomy, and course of unilateral neglect in an acute stroke. Neurology, Vol. 63, No. 3, 468-474, 2004.

[19] G. Kerkhoff. Spatial neglect in humans, Progress in Neurobiology Vol. 63, 1-27, 2001.

[20] E. A. Zillmer, M.V. Spiers, W. C. Culbertson WC. Principles of neuropsychology (2nd ed.), Cengage, Belmont, CA, 2007.
[21] M. D. Lezak, D. B. Howieson, D. W. Loring, H. J. Hannay, J. S. Fischer. Neuropsychological assessment (4th ed.). Oxford University Press, New York, 2012.

[22] R. Wenman, A. Bowen, R. Tallis, E. Gardener, S. Cross, D. Niven D. Use of randomized single case experimental design to evaluate therapy for unilateral neglect, Neuropsychological Rehabilitation, Vol.13, No.4, 441-459, 2003.

[23] J. C. Adair, A. M. Barrett. Spatial neglect clinical and neuroscience review: A wealth of information on the poverty of attention, Annals of the New York Academy of Sciences, Vol. 1142, 21-43, 2008

[24] G. Kerkhoff, T. Schenk. Rehabilitation of neglect: an update, Neuropsychologia, Vol. 50, 1072-1079, 2012

[25] M. Rizzo, B. A. Kellison. Eyes, brains, and autos, Archives of Ophthalmology, Vol. 122, No. 4, 641-647, 2004.

[26] S. E. Starkstein, H. S. Mayberg, M. L. Berthier, P. Fedoroff, T. R. Price, R. F. Dannals, H. N. Wagner, R. Leiguarda, R. G. Robinson. Mania after brain injury: neuroradiological and metabolic findings, Annals of Neurology, Vol. 27, No. 6, 652-659, 1990.

[27] T. Cassidy, S. Lewis, C. S. Gray. Recovery from visuospatial neglect in stroke patients, Journal of Neurology, Neurosurgery and Psychiatry, Vol. 64, No. 4, 555-557, 1998.

[28] L. Pizzamiglio, A. Judica, C. Razzano, P. Zoccolotti P. Toward a comprehensive diagnosis of visual-spatial disorders in unilateral brain-damaged patients, Psychological Assessment, Vol. 5, 199-218, 1989.

[29] L. J. Buxbaum, M. K. Ferraro, T. Veramonti, A. Farne, J. Whyte, E. Ladavas, F. Frassinetti, H. B. Coslett HB. Hemispatial neglect: Subtypes, neuroanatomy, and disability, Neurology, Vol. 62, No. 5, 749-756, 2004.

[30] H. O. Karnath. Spatial attention systems in spatial neglect, Neuropsychologia, Vol. 75, 61-73, 2015.

[31] M. Albert. A simple test of visual neglect, Neurology, Vol. 23, 658-664, 1973

[32] L. Costa, H. Vaughan, M. Horwitz, W. Ritter W. Patterns of behavioural deficit associated with visual spatial neglect, Cortex, Vol. 52, 242-263, 1969.

[33] S. Paolucci, G. Antonucci, M. G. Grasso, L. Pizzamiglio L. The role of unilateral spatial neglect in rehabilitation of right brain-damaged ischemic stroke patients: a matched comparison, Archives of Physical Medicine and Rehabilitation, Vol. 82, 743-749, 2001.

[34] J. Luauté, P. Halligan, G. Rode, Y. Rossetti, D. Boisson. Visuo-spatial neglect: a systematic review of current interventions and their effectiveness, Neuroscience and Biobehavioral Reviews, Vol. 30, 961-982, 2006.

[35] G. Vallar. Spatial neglect, Balint-Homes' and Gerstmann's syndromes, and other spatial disorders, CNS Spectrums, Vol. 12, No. 7, 527-536, 2007.

[36] R. Jepson, K. Despain, D. C. Keller. Unilateral neglect: Assessment in nursing, Journal of Neuroscience Nursing Vol. 40, No. 3, 142-149, 2008.

[37] J. Smith, D. Hebert, D. Reid D. Exploring the effects of virtual reality on unilateral neglect caused by stroke: Four case studies, Technology and Disability, Vol.19, 29-40, 2007. 
[38] D. F. Edwards, M. G. Hahn, C. M. Baum, M. S. Perlmutter, C. Sheedy, A. W. Dromerick. Screening patients with stroke for rehabilitation needs: validation of the post-stroke rehabilitation guidelines, Neurorehabilitation and Neural Repair, Vol. 20, No. 1, 42-48, 2006.

[39] A. Farné, L. J. Buxbaum, M. Ferraro, F. W. Frassinetti, J. Whyte, T. Veramonti, V. Angeli, H. B. Coslett, E. Làdavas E. Patterns of spontaneous recovery of neglect and associated disorders in acute right brain-damaged patients, Journal of Neurology and Neurosurgery Psychiatry, Vol. 75, 1401-1410, 2002.

[40] J. Driver, J. B. Mattingley. Parietal neglect and visual awareness, Nature Neuroscience, Vol.1, 17-22, 1998.

[41] M. Husain. Hemineglect, Scholarpedia, Vol. 3, No. 2, 3681, 2008.

[42] E. A. Zillmer, M. V. Spiers. Principles of neuropsychology, Wadsworth, Belmont, California, 2001.

[43] J. Danckert, S. Ferber. Revisiting unilateral neglect, Neuropsychologia, Vol. 44, 987-1006, 2006.

[44] L. Swan L. Unilateral spatial neglect, Physical Therapy, Vol. 81, 1572-1580, 2001.

[45] M. Jehkonen, M. Laihosalo, J. E. Kettunen. Impact of neglect on functional outcomes after stroke - A review of methodological issues and recent research findings, Restorative Neurology and Neuroscience, Vol. 24, 209-215, 2006.

[46] A. E. Hillis, S. Chang, J. Heidler-Gary, M. Newhart, J. T. Kleinman, C. Davis, et al. Neural correlates of modality-specific spatial extinction, Journal of Cognitive Neuroscience, Vol. 18, 1889-1898, 2006.

[47] E. Ladavas, G. Zeloni. Eye movements and orienting of attention in patients with visual neglect, Journal of Cognitive Neuroscience, Vol. 9, No. 1, 67-74, 1997.

[48] J. B. Mattingley, M. Husain. Motor role of human inferior parietal lobe revealed in unilateral neglect patients, Nature, Vol. 392, 179-182, 1998.

[49] M. M. Mesulam. Attention, confusional states, and neglect. In M. M. Mesulam (Ed.), Principles of behavioral neurology (pp. 125-168). Davis, Philadelphia, Pennsylvania, 1985.

[50] D. Punt, J. Riddoch J. Motor neglect: Implications for movement and rehabilitation following stroke, Disability and Rehabilitation, Vol. 28, 857-864, 2006.

[51] A. E. Hillis, M. Newhart, J. Heidler, E. B. Marsh, P. Barker, M. Degoankar M. The neglected role of the right hemisphere in spatial representation of words for reading, Aphasiology, Vol. 19, 225-238, 2005.

[52] G. Rode, T. Klos, S. Jacquin, Y. Rossetti, L. Pisella. Neglect and prism adaptation: A new therapeutic tool for spatial cognition disorders, Restorative Neurology and Neuroscience, Vol. 24, 347-356, 2006.

[53] P. W. Halligan, J. C. Marshall. The history and clinical presentation of neglect. In I. H. Robertson, and J. C. Marshall (Eds.), Unilateral neglect: Clinical and experimental studies (pp. 3-25), Lawrence Erlbaum, Hillsdale, New Jersey, 1993.

[54] K. M. Heilman, R. T. Watson, E. Valenstein. Neglect and related disorders. In K. M. Heilman, and E. Valenstein (Eds.), Clinical neuropsychology (5th ed., pp. 296-348). Oxford, New York, 2012.

[55] S. Primativo, L. S. Arduino, M. De Luca, R. Daini, M. Martelli. Neglect dyslexia: A matter of "good looking", Neuropsychologia, Vol. 51, 2109-2119, 2013.

[56] P. Vuilleumier, S. Schwartz. Emotional facial expressions capture attention, Neurology, Vol. 56, 153-158, 2001.

[57] A. W. Young, E. H. de Haan, F. Newcombe, D. C. Hay. Facial neglect, Neuropsychologia, Vol. 28, 391-415, 1990.

[58] R. Eramudugolla, D. R. F. Irvine, J. B. Mattingley. Association between auditory and visual symptoms of unilateral spatial neglect,. Neuropsychologia, Vol. 45, 2631-2637, 2007.

[59] L. Spierer, R. Meuli, S. Clarke. Extinction of auditory stimuli in hemineglect: Space versus ear, Neuropsychologia, Vol. 45, 540-551, 2007.

[60] N. Soroker, N. Calamaro, J. Glicksohn, M. S. Myslobodsky. Auditory inattention in right-hemisphere-damaged patients with and without visual neglect, Neuropsychologia, Vol. 35, No. 3, 249-256, 1997.

[61] P. Appelros, G. M. Karlsson, S. Hennerdal S. Anosognosia versus unilateral neglect: Coexistence and their relations to age, stroke severity, lesion site and cognition, European Journal of Neurology, Vol. 14, 54-59, 2007.

[62] C. Morin, S. Thibierge, M. Perrigot. Right brain damage, body image, and language: A psychoanalytic perspective, Journal of Mind and Behavior, Vol. 22, 69-90, 2001.

[63] A. M. Barrett, L. J. Buxbaum, H. B. Coslett, E. Edwards, K. M. Heilman, A. E. Hillis, et al. Cognitive rehabilitation interventions for neglect and related disorders: moving from bench to bedside in stroke patients, Journal of Cognitive Neuroscience, Vol.18, 1223-1236, 2006.

[64] P. Appelros, G. M. Karlsson, A. Seiger,I. Nydevi I. Neglect and anosognosia after first-ever stroke: Incidence and relationship to disability, Journal of Rehabilitative Medicine, Vol. 34, 215-220, 2002.

[65] B. Vahlber, K. Hellström K. Treatment and assessment of neglect after stroke- from a physiotherapy perspective: a systematic review, Advances in Physiotherapy, Vol. 10, $178-187,2008$

[66] A. Parton, P. Malhotra, M. Husain. Hemispatial neglect, Journal of Neurology and Neurosurgical Psychiatry, Vol. 75, 13-21, 2004.

[67] G. W. Humphreys, M. J. Riddoch. Interactive attentional systems and uni-lateral visual neglect. In I. Robertson, J. C. Marshall (Eds.), Unilateral Neglect. Lawrence Erlbaum Associates, Hove, 139-167, 1993.

[68] G. Rizzolatti, A. Berti. Neural mechanisms of spatial neglect. In I. H. Robertson, and J. C. Marshall (Eds.), Unilateral neglect: Clinical and experimental studies (pp. 87-105), Erlbaum, Hillsdale, New Jersey, 1993.

[69] P. Appelros, I. Nydevik, G. M. Karlsson, A. Thorwalls, A. Seiger. Recovery from unilateral neglect after right-hemisphere stroke, Disability and Rehabilitation, Vol. 26, No. 8, 471-477, 2004. 
[70] J. E. Goff. Assessing unilateral neglect in acute care: Raising clinician awareness, OT Practice, Vol. 15, No. 22, 14-17, 2010 .

[71] A. Menon-Nair, N. Korner, T. Ogourtsova. Occupational therapists' identification, assessment, and treatment of unilateral spatial neglect during stroke rehabilitation in Canada, Stroke, Vol. 38, 2556-2562, 2007.

[72] J. Marshall. Line bisection and neglect severity, Brain and Cognition, Vol. 38, 275-280, 1998.

[73] M. C. Mozer, P. W. Halligan. The end of the line for a brain-damaged model of unilateral neglect, Journal of Cognitive Neuroscience, Vol. 9, No. 2, 171-190, 1997.

[74] V. W. Mark, K. M. Heilman. Diagonal spatial neglect, Journal of Neurology, Neurosurgery and Psychiatry, Vol. 65, No. 3, 348-352, 1998.

[75] I. Keller, K. Volkening, R. Garbacenkaite. Stimulus-dependent modulation of visual neglect in a touch screen cancellation task, Neuropsychology, Vol. 29, No. 3, 417-420, 2015.

[76] S. Ishiai, K. Seki, Y. Koyama, Y. Izumi. Disappearance of unilateral spatial neglect following a simple instruction, Journal of Neurology, Neurosurgery and Psychiatry, Vol. 63, No. 1, 23-27, 1997.

[77] S. Ishiai, M. Sugishita, T. Ichikawa, S. Gono, S. Watabiki. Clock-Drawing Test and unilateral spatial neglect, Neurology, Vol. 43, No.1, 106-110, 1993.

[78] M. Jehkonen, J. P. Ahonen, P. Dastidar, A. M. Koivisto, P. Laippala, J. Vilkki. How to detect visual neglect in acute stroke, Lancet, Vol. 351, 727-728, 1998.

[79] B. Wilson, J. Cockburn, P. Halligan. Development of a behavioral test of visuo-spatial neglect, Archives of Physical Medicine and Rehabilitation, Vol. 68, 98-102, 1987.
[80] R. L. Schwartz, A. M. Barrett, M. Kim, K. M. Heilman. Ipsilesional intentional neglect and the effect of cueing. Neurology, Vol. 53, 2017-2022, 1999.

[81] P. Azouvi, S. Olivier, G. De Montety, S. C. Louis-Dreyfus, A. Lousi-Dreyfus, L. Tesio. Behavioral assessment of unilateral neglect: Study of the psychometric properties of the Catherine Bergego Scale, Archives of Physical Medicine and Rehabilitation, Vol. 84, 51-57, 2003.

[82] L. Pizzamiglio, C. Guariglia, G. Antonucci, P. Zoccolotti. Development of rehabilitative program for unilateral neglect, Restorative Neurology and Neuroscience, Vol. 24, 337-345, 2006.

[83] J. H. Cauraugh, N. Lodha, S. K. Naik, J. J. Summers. Bilateral movement training and stroke motor recovery progress: a structured review and meta-analysis, Human Movement Science, Vol. 29, No. 5, 853-870, 2010.

[84] C. Norrbrink. Transcutaneous electrical nerve stimulation for treatment of spinal cord injury neuropathic pain, Journal of Rehabilitation Research and Development, Vol. 46, No. 1, 85-93, 2009.

[85] D. Skeil, A. C. Thorpe. Transcutaneous electrical nerve stimulation in the treatment of neurological patients with urinary symptoms. British Journal of Urology International, Vol. 88, No. 9, 899-908, 2001.

[86] R. M. Dubinsky, J. Miyasaki. Assessment: efficacy of transcutaneous electric nerve stimulation in the treatment of pain in neurologic disorders (an evidence-based review): report of the Therapeutics and Technology Assessment Subcommittee of the American Academy of Neurology, Neurology, Vol. 74, No. 2, 173-176, 2009.

[87] C. L. Fink. Stroke rehabilitation. 4. Stroke outcome and psychosocial consequences, Archives of Physical Medicine and Rehabilitation, Vol. 80, S21-S26, 1999. 